\title{
Clinical characteristics and risk factors for mortality in patients with coronavirus disease 2019 in intensive care unit: a single- center, retrospective, observational study in China
}

\author{
Fangfang Sai ${ }^{1 \#}$, Xiaolei Liu ${ }^{2 \#}$, Lanyu $\mathrm{Li}^{2}$, Yan $\mathrm{Ye}^{3}$, Changqing Zhu ${ }^{2}$, Ying Hang ${ }^{2}$, Conghua Huang ${ }^{2}$, Lei Tian ${ }^{2}$, Huan Huang ${ }^{2}$, \\ Xinhui $\mathrm{Xu}^{2}$ \\ ${ }^{1}$ Department of Geriatrics, Renji Hospital, School of Medicine, Shanghai Jiaotong University, Shanghai, China; ${ }^{2}$ Department of Emergency \\ Medicine, Renji Hospital, School of Medicine, Shanghai Jiaotong University, Shanghai, China; ${ }^{3}$ Department of Rheumatology Renji Hospital, \\ School of Medicine, Shanghai Jiaotong University, Shanghai, China \\ Contributions: (I) Conception and design: H Huang, X Xu; (II) Administrative support: C Zhu; (III) Provision of study materials or patients: H \\ Huang, L Tian, Y Hang, C Huang; (IV) Collection and assembly of data: X Liu, F Sai; (V) Data analysis and interpretation: X Liu, L Li, Y Ye; (VI) \\ Manuscript writing: All authors; (VII) Final approval of manuscript: All authors. \\ "These authors contributed equally to this work. \\ Correspondence to: Huan Huang, MD; Xinhui Xu, MD. Department of Emergency Medicine, Renji Hospital, School of Medicine, Shanghai Jiaotong \\ University, 160\# Pujian Rd, Pudong New District, Shanghai 200127, China. Email: renjihuanghuan@163.com; xinhui_72@hotmail.com.
}

Background Coronavirus disease 2019 (COVID-19) is a potentially life-threatening contagious disease which has spread all over the world. Risk factors associated with the clinical outcomes of COVID-19 pneumonia in intensive care unit (ICU) have not yet been well determined.

Methods: This was a retrospective, single-centered, observational study, in which 47 patients with confirmed COVID-19 were consecutively enrolled from February 24 to April 5, 2020. The patients were registered from the ICU of Leishenshan Hospital in Wuhan, China. Clinical characteristics and outcomes were collected and compared between survivors and non-survivors. Multivariable logistic regression was performed to analyze the risk factors of death in patients with COVID-19.

Results: The study cohort included 47 adult patients with an average age of $70.55 \pm 12.52$ years, and $30(63.8 \%)$ patients were men. Totally 15 (31.9\%) patients died. When compared to survivors, nonsurvivors showed a higher proportion of septic shock [6 (40\%) patients vs. 3 (9.4\%) patients], disseminated intravascular coagulation [3 (21.4\%) vs. 0], and had higher score of APACHE II (25.07 \pm 8.03 vs. 15.56 \pm 5.95$)$, CURB-65 \{3 [2-4] vs. 2 [1-3]\}, Sequential Organ Failure Assessment (SOFA) \{7 [5-9] vs. 3 [1-6]\}, higher level of D-dimer $\{5.74[2.32-18]$ vs $2.05[1.09-4.00]\}$ and neutrophil count $\{9.4$ [7.68-14.54] vs. 5.32 [3.85-9.34]\}. SOFA score (OR 1.47; 95\% CI: 1.01-2.13; P=0.0042) and the lymphocyte count (OR 0.02; 95\% CI: 0.00-0.86; $\mathrm{P}=0.042)$ on admission were independently risk factors for mortality. Patients with higher lymphocyte count $\left(>0.63 \times 10^{9} / \mathrm{L}\right)$ and lower SOFA score $(\leq 4)$ on admission had a significantly better prognosis than those with lower lymphocyte count $\left(\leq 0.63 \times 10^{9} / \mathrm{L}\right)$ and higher SOFA score $(>4)$ in overall survival.

Conclusions: Higher SOFA score and lower lymphocyte count at admission were connected with poor prognosis of patients with COVID-19 in ICU. Lymphocyte count may serve as a promising prognostic biomarker.

Keywords: Coronavirus disease 2019 (COVID-19); mortality; lymphocyte count; intensive care unit (ICU)

Submitted Aug 09, 2020. Accepted for publication Dec 17, 2020.

doi: $10.21037 /$ apm-20-1575

View this article at: http://dx.doi.org/10.21037/apm-20-1575 


\section{Introduction}

In December 2019, China reported to the World Health Organization (WHO) cases of pneumonia in Wuhan, Hubei Province, China, caused by a novel coronavirus, severe acute respiratory syndrome coronavirus 2 (SARS-CoV-2) (1). In February 2020, its associated disease was designated coronavirus disease 2019 (COVID-19), and was declared a public health emergency of international concern by the World Health Organization (WHO) (2). Globally, as of 13 November 2020, there have been 52,487,476 confirmed cases of COVID-19, including 1,290,653 deaths, reported to $\mathrm{WHO}(3)$.

According to the WHO-China Joint Mission on COVID-19 report, $13.8 \%$ of patients with laboratoryconfirmed COVID-19 developed severe disease and $6.1 \%$ required intensive care (4). In a previous study of 1099 patients with laboratory-confirmed COVID-19 from 552 hospitals in 30 Chinese provinces, $5.0 \%$ were admitted to the intensive care unit (ICU), 2.3\% underwent invasive mechanical ventilation, and $1.4 \%$ died (5). Two subsequent studies demonstrated that $23-26 \%$ of COVID-19 cases required admission to the ICU and the mortality rate varied from $4.3-11 \%$, most of the patients in these studies were still in hospital at the time of the manuscripts were submitted $(6,7)$. Early studies in China revealed that patients with severe SARS-CoV-2 pneumonia who were admitted to the ICU had a high mortality rate $(8,9)$, while a number of large studies from Europe reported ICU mortality rates of $26-32 \%(10-12)$.

Several epidemiological characteristics and clinical manifestations have been associated with COVID-19. For example, older patients with comorbidities were found to have a high risk of developing acute respiratory distress syndrome (ARDS) and death $(8,13)$. A recent study from two designated hospitals in Wuhan demonstrated that older age, a higher Sequential Organ Failure Assessment (SOFA) score on admission, and elevated levels of baseline D-dimer $(>1 \mu \mathrm{g} / \mathrm{L})$ were associated with an increased rate of deaths during hospitalization (14). However, few studies evaluated the risk factors of COVID-19-related death in the ICU. Thus, in this retrospective study, we aimed to explore the risk factors of death by investigating clinical features, laboratory characteristics and short-term outcomes of patients with severe cases of COVID-19 from a designated COVID-19 hospital in Wuhan.

We present the following article in accordance with the STROBE reporting checklist (available at http://dx.doi. org/10.21037/apm-20-1575).

\section{Methods}

\section{Study design}

All procedures described here have been approved by the ethics committee of Leishenshan Hospital. This study was retrospectively conducted at Leishenshan Hospital (Wuhan, China), which was a designated hospital with 1,600 beds, including two ICUs (A and B). All critical patients with diagnosed COVID-19 admitted to B-ICU from February 24 to April 52020 were consecutively enrolled. Critically ill patients with COVID-19 were defined as those complicated with at least one of the following: respiratory failure requiring mechanical ventilation, septic shock, other organ failure requiring ICU monitoring and treatment (15). The diagnosis of COVID-19 pneumonia was made on the basis of WHO interim guidance (16). The primary outcome was 60-day mortality after ICU admission.

\section{Data collection}

All the patients' electronic medical records, nursing records, laboratory findings, and radiological examinations were reviewed. We collected data including demographics, underlying chronic diseases (chronic heart disease, chronic pulmonary disease, diabetes, malignancy, malnutrition, chronic liver disease and chronic kidney disease), laboratory findings, chest computed tomographic scans, treatment (including antiviral therapy, antibiotics, corticosteroid therapy, oxygen support, renal replacement therapy and extracorporeal membrane oxygenation), clinical complications [septic shock, acute respiratory distress syndrome (ARDS), secondary infection, acute kidney injury and acute cardiac injury] and outcome data during the hospital admission. The CURB-65, Sequential Organ Failure Assessment (SOFA), and Acute Physiology and Chronic Health Evaluation II scores (APACHE II) scores were evaluated on the day of ICU admission.

Septic shock was defined as sepsis associated with systemic inflammatory response syndrome, organ dysfunction and persistent hypotension after volume replacement (17). Acute kidney injury was defined by an abrupt decrease in kidney function that includes, but is not limited to, acute renal failure (18). Acute cardiac injury was diagnosed when serum levels of cardiac biomarkers (e.g., high-sensitive cardiac troponin I) exceed the 99th percentile upper reference limit 
or there were new abnormalities in electrocardiography and echocardiography (19). Acute respiratory distress syndrome (ARDS) was diagnosed according to the Berlin Definition (20). Secondary infection was diagnosed when the patients developed clinical manifestations of nosocomial pneumonia or bacteraemia, and a new positive pathogen was cultured from the lower respiratory tract or blood sample $\geq 48 \mathrm{~h}$ after admission (21).

The study was conducted in accordance with the Declaration of Helsinki (as revised in 2013). The study was approved by the ethics committee of Leishenshan Hospital, which was completely managed by the Renji Hospital (Shanghai Jiaotong University School of Medicine) (NO.: [2020]023) and individuals consent for this retrospective analysis was waived.

\section{Statistical analysis}

Continuous variables were presented as median (IQR) or mean \pm standard deviation. Categorical variables were presented as number (\%). The Mann-Whitney $U$ test was used to compare continuous variables. Categorical variables were compared using the chi-square test or Fisher's exact test. Variables that were significant at $\mathrm{P}<0.1$ in the univariate analysis were included in the forward stepwise multivariate logistic regression model. The receiver operating characteristic (ROC) curve was drawn to calculate the area under the curve (AUC), to assess the predictive performance of the lymphocyte count and SOFA at admission for outcome. Patient survival according to appropriated cutoff value of the lymphocyte count and SOFA score at admission were determined using the logrank test and displayed using Kaplan-Meier curves. A P value less than 0.05 were considered statistically significant. All data were analyzed using the IBM SPSS Statistics for Windows (version 19.0). The strength of any association was evaluated by calculating odds ratio (OR) and $95 \%$ confidence interval (CI).

\section{Results}

\section{Baseline characteristics}

By April 5, 2020, 52 patients with COVID-19 had been admitted to the B-ICU of Wuhan Leishenshan hospital. After excluding five patients without confirmed SARSCoV-2 RNA or without complete medical information, we finally included 47 inpatients in this study (Table 1).
The mean age was $70.55 \pm 12.52$ years (range, $38-93$ years). Thirty $(63.8 \%)$ patients were male. Comorbidities were present in $40(68.1 \%)$ patients, with hypertension the most common comorbidity $[\mathrm{n}=25(53.2 \%)]$, followed by diabetes $[\mathrm{n}=18(38.3 \%)]$, and chronic kidney disease $[\mathrm{n}=15$ (31.9\%)]. The average APACHE II score of all patients was $18.6 \pm 7.79$. A total of $34(72.3 \%)$ patients were found to have bilateral infiltrates on radiographic imaging.

\section{Differences of clinical characteristics between survivors and non-survivors}

The median SOFA score in non-survivors (7, IQR 5-9) was much higher than that of survivors (3, IQR: 1-6), whereas the median lymphocyte count of ICU patients was higher in survivors $\left(0.77 \times 10^{9} / \mathrm{L}\right.$, IQR: $\left.0.54 \times 10^{9}-1.29 \times 10^{9}\right)$ than nonsurvivors $\left(0.54 \times 10^{9} / \mathrm{L}\right.$, IQR: $\left.0.26 \times 10^{9}-0.63 \times 10^{9}\right)$. Compared with survivors, non-survivors had significantly higher levels of C-reactive protein, procalcitonin, interleukin (IL)-6 and IL-1B, and were more likely to develop septic shock [6 (40\%) vs. 3 (9.4\%), respectively], and disseminated intravascular coagulation [3 (21.4\%) vs. 0, respectively].

\section{Clinical outcomes}

For the primary outcome, 15 (31.9\%) of 47 patients died in our study. ARDS ( $\mathrm{n}=12 ; 25.5 \%)$, acute cardiac injury $(\mathrm{n}=12 ; 25.5 \%)$, and acute kidney injury $(\mathrm{n}=10$; $21.3 \%$ ) were frequently observed in ICU patients. Three patients developed bloodstream infections of Klebsiella pneumoniae, Staphylococcus aureus and Enterococcus faecalis during hospitalization. Invasive mechanical ventilation was performed in $13(27.7 \%)$ patients, of whom nine died. Median durations from admission to invasive mechanical ventilation and from invasive mechanical ventilation to death were 6.0 (range, 2.00-11.00) days, and 5.54 5.98 days respectively. Two patients were rescued by extracorporeal membrane pulmonary oxygenation, of whom one died. Forty-five $(95.7 \%)$ patients received intravenous antibiotics and $11(23.4 \%)$ received systematic corticosteroids. Three patients $(6.4 \%)$ received plasma treatment from patients who recovered from COVID-19, all of whom survived (Table 1).

\section{Risk factors of mortality}

Univariate analysis revealed the following variables were associated with death: the APACHE II score, the CURB65 score, the SOFA score, the presence of ARDS, chronic 
Table 1 Comparison of clinical characteristics between COVID-19 survivors and non-survivors

\begin{tabular}{|c|c|c|c|c|}
\hline Demographic data and clinical characteristics & Total $(n=47)$ & Survivors $(n=32)$ & Non-survivors $(n=15)$ & $P$ \\
\hline Age & $70.55 \pm 12.52$ & $69.67 \pm 12.91$ & $70.64 \pm 12.33$ & 0.811 \\
\hline \multicolumn{5}{|l|}{ Underlying diseases } \\
\hline Comorbidity, n (\%) & $40(68.1)$ & $26(81.3)$ & $14(29.8)$ & 0.404 \\
\hline Diabetes mellitus, $\mathrm{n}(\%)$ & $18(38.3)$ & $12(37.5)$ & $6(40.0)$ & 0.559 \\
\hline Chronic kidney disease, n (\%) & $15(31.9)$ & $14(46.7)$ & $1(7.1)$ & 0.025 \\
\hline Chronic liver disease, n (\%) & $6(12.8)$ & $3(9.4)$ & $3(20.0)$ & 0.367 \\
\hline Bilateral involvement of chest radiographs, $\mathrm{n}(\%)$ & $34(72.3)$ & $26(81.3)$ & $8(53.3)$ & 0.079 \\
\hline APACHEII & $18.6 \pm 7.97$ & $15.56 \pm 5.95$ & $25.07 \pm 8.03$ & 0.406 \\
\hline CURB-65 & $2(1-3)$ & $2(1-3)$ & $3(2-4)$ & 0.001 \\
\hline SOFA score & $5(2-7)$ & $3(1-6)$ & $7(5-9)$ & 0.000 \\
\hline \multicolumn{5}{|l|}{ Laboratory finding } \\
\hline White-cell count, $\times 10^{9} / \mathrm{L}$ & $8.25(5.60-11.98)$ & $7.20(5.16-11.32)$ & $10.70(8.50-16.53)$ & 0.087 \\
\hline Neutrophil count, $\times 10^{9} / \mathrm{L}$ & $6.67(3.97-10.48)$ & $5.32(3.85-9.34)$ & $9.40(7.68-14.54)$ & 0.027 \\
\hline Lymphocyte count, $\times 10^{9} / \mathrm{L}$ & $0.77(0.54-1.29)$ & $0.99(0.66-1.56)$ & $0.54(0.26-0.63)$ & 0.000 \\
\hline Total bilirubin, U/L & $10.00(6.80-16.10)$ & $9.80(6.20-15.73)$ & $11.00(6.50-20.33)$ & 0.741 \\
\hline Lactate dehydrogenase, $\mathrm{mmol} / \mathrm{L}$ & $286.00(224.00-460.00)$ & $263.5(211.25-367.75)$ & $424.5(271.25-551.25)$ & 0.043 \\
\hline Aspartate aminotransferase, U/L & $25.00(12.00-40.00)$ & $22.50(10.00-38.50)$ & $32.00(15.75-56.00)$ & 0.664 \\
\hline Glucose, mmol/L & $6.44(5.06-8.33)$ & $6.00(4.95-7.64)$ & $7.70(5.49-12.35)$ & 0.077 \\
\hline Creatinine, $\mu \mathrm{mol} / \mathrm{L}$ & $86.70(57.40-157.40)$ & $87.40(59.83-194.00)$ & $107.50(57.45-182.55)$ & 0.706 \\
\hline D-dimer, mg/mL & $2.68(1.50-5.83)$ & $2.05(1.09-4.00)$ & $5.74(2.32-18.00)$ & 0.008 \\
\hline Prothrombin time, s & $12.4(11.5-14.3)$ & $12.30(11.50-13.78)$ & $13.20(11.70-16.00)$ & 0.288 \\
\hline Activated partial thromboplastin time, s & $32.40(28.2-40.4)$ & $32.20(28.38-40.30)$ & $32.70(28.10-46.80)$ & 0.945 \\
\hline Troponin I, ng/mL & $0.04(0.02-0.08)$ & $0.0(0.01-0.07)$ & $0.0(0.02-0.10)$ & 0.614 \\
\hline BNP, pg/mL & $123.00(32.89-563.00)$ & $169.50(28.98-745.75)$ & $114.50(63.57-380.75)$ & 0.873 \\
\hline $\mathrm{IL}-1 \beta, \mathrm{pg} / \mathrm{mL}$ & $4.0(2.0-5.2)$ & $3.0(2.0-4.5)$ & $5.6(4.0-25.7)$ & 0.026 \\
\hline
\end{tabular}

Table 1 (continued) 
Table 1 (continued)

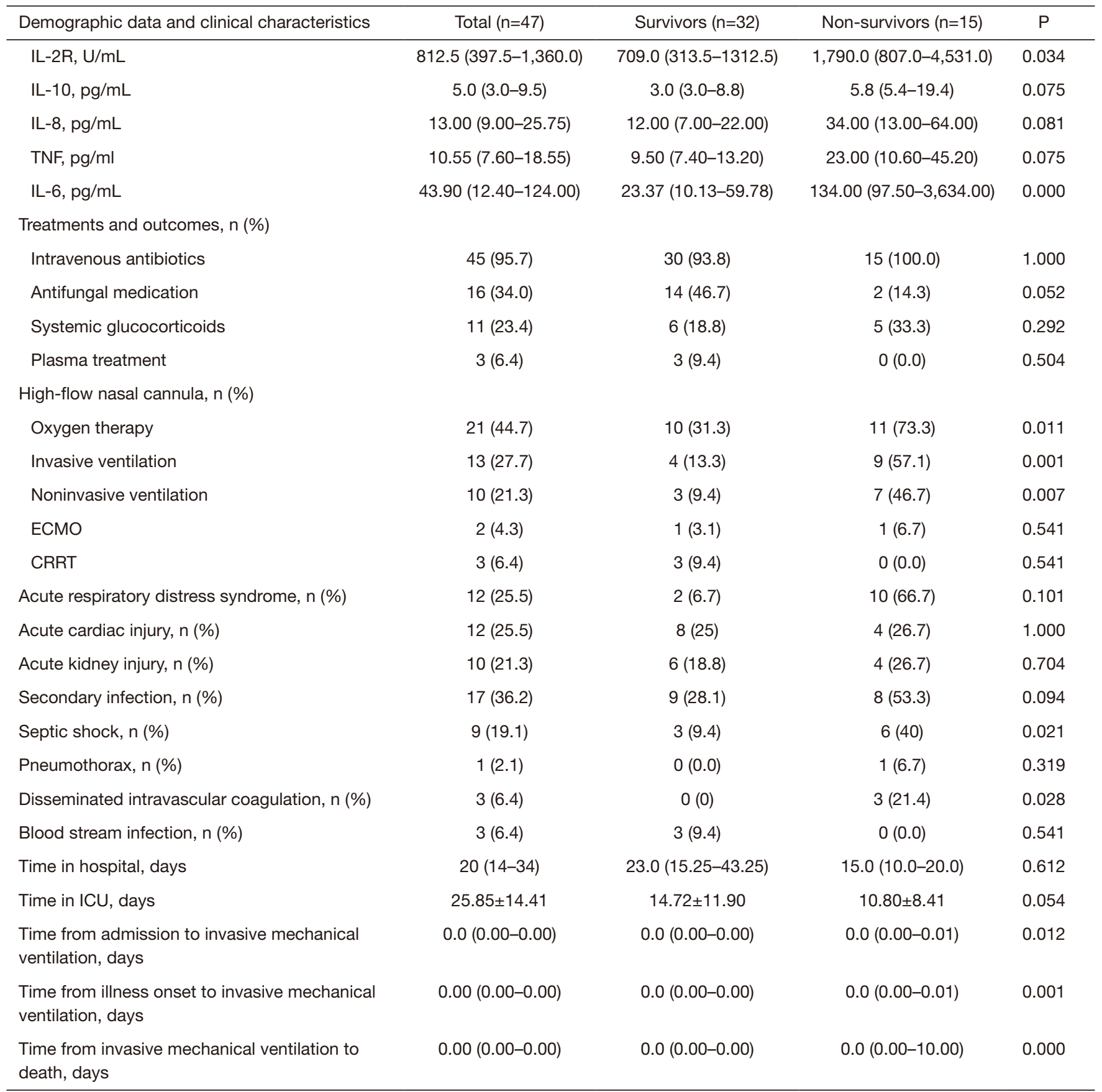

Continuous variables conforming to the normal distribution were presented by mean \pm standard deviation, otherwise by the median (IQR). COVID-19, coronavirus disease 2019; APACHE II, Acute Physiology and Chronic Health Evaluation II; CURB-65, Confusion, Respiratory rate, Blood pressure, Age $\geq 65$ years old; SOFA, Sequential Organ Failure Assessment; PCT, procalcitonin; BNP, brain natriuretic peptide; IL, interleukin; ECMO, extracorporeal membrane pulmonary oxygenation; CRRT, Continuous Renal Replacement Therapy. 
Table 2 Risk factors associated with in-hospital death

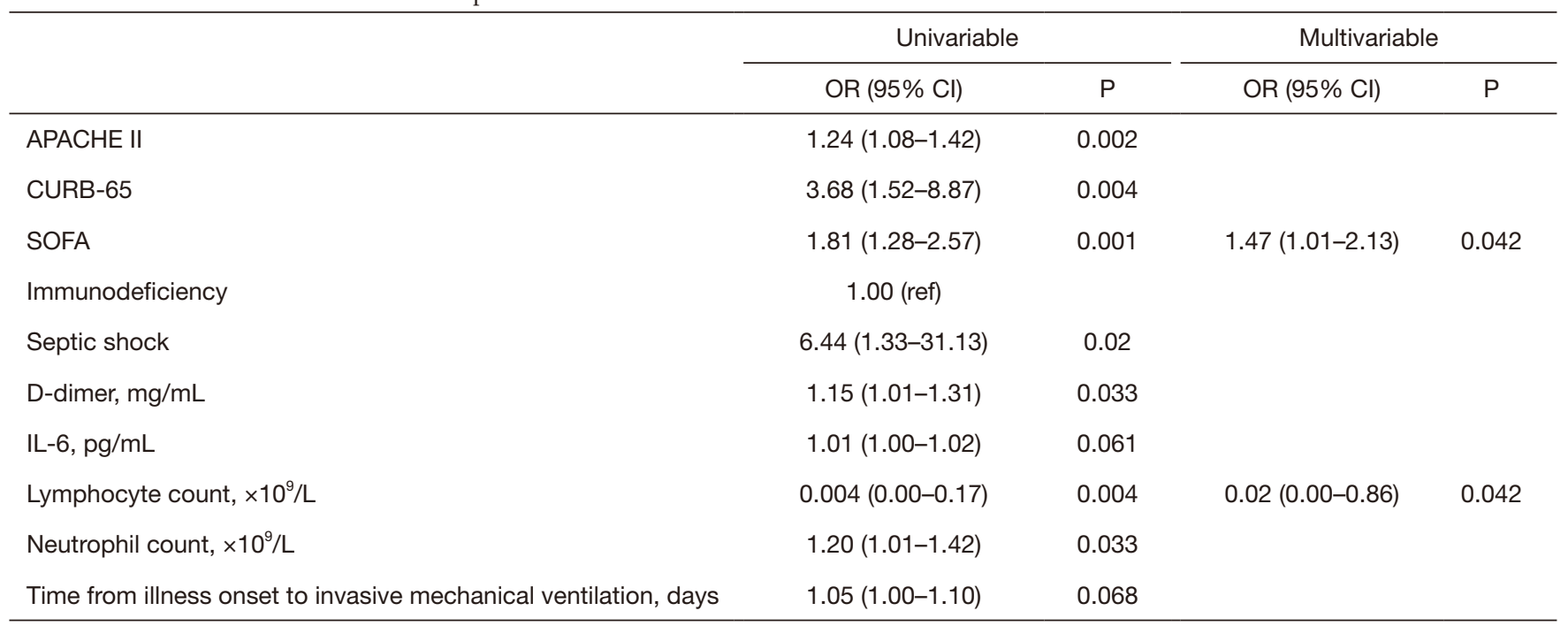

APACHE II, Acute Physiology and Chronic Health Evaluation II; CURB-65, Confusion, Respiratory rate, Blood pressure, Age $\geq 65$ years old; SOFA, Sequential Organ Failure Assessment; IL, interleukin.

heart disease, and septic shock, the lymphocyte count, and the neutrophil count (Table 2). These were investigated via multivariate logistic regression, which showed that the SOFA score $(\mathrm{OR}=1.47,95 \% \mathrm{CI}: 1.01-2.13, \mathrm{P}=0.04)$ and lymphocyte count at admission ( $\mathrm{OR}=0.02,95 \% \mathrm{CI}$ : 0.00 $0.86, \mathrm{P}=0.04$ ) were independent factors related to mortality (Table 2).

\section{Predictive value of lymphocyte count and SOFA score for survival}

Compared with non-survivors, the lymphocyte count at admission was significantly higher in survivors. The lymphocyte count was lowest at baseline and improved during hospitalization among survivors, whereas it decreased continuously until death among non-survivors (Figure 1).

Lymphocyte count was shown to be significantly associated with survival (AUC 0.865; 95\% CI: 0.375-0.781; $\mathrm{P}<0.0001$ ), with the optimal cutoff value identified as $0.63 \times 10^{9} / \mathrm{L}$. Thus, patients were divided into two groups according to the lymphocyte count. Figure 2 shows the survival curve of the two groups (the lymphocyte count $>0.63 \times 10^{9} / \mathrm{L} v s . \leq 0.63 \times 10^{9} / \mathrm{L}$ ) by the Kaplan-Meier analysis. The log-rank test shows patients with a higher lymphocyte count $\left(>0.63 \times 10^{9} / \mathrm{L}\right)$ on admission had a significantly better prognosis than those with a lower lymphocyte count $\left(\leq 0.63 \times 10^{9} / \mathrm{L}\right)$ in terms of overall survival $(\mathrm{P}=0.001)$.

The SOFA score was also shown to be significantly associated with survival (AUC 0.860; 95 \% CI: 0.728-0.944; $\mathrm{P}<0.0001)$, with the optimal cutoff value identified as 4. Thus, patients could be also divided into two groups according the SOFA score. Figure 3 shows the survival curve of the two groups (the SOFA score $\leq 4 v s .>4$ ) by the KaplanMeier analysis. The log-rank test shows patients with a lower SOFA score $(\leq 4)$ on admission had a significantly better prognosis than those with a higher SOFA score $(>4)$ in terms of overall survival $(\mathrm{P}=0.001)$.

\section{Discussion}

In our study, the mortality rate of COVID-19 patients was $31.9 \%$. Our findings demonstrated that survivors and nonsurvivors differed with respect to clinical characteristics and indicators of inflammation. Higher SOFA scores and lower lymphocyte counts at baseline were associated with an increased in-hospital death rate. More importantly, we found that the lymphocyte count on admission may serve as a predictive biomarker for survival in severe COVID-19 cases.

Our observed fatality of $31.9 \%$ was lower than that reported in other ICUs in Wuhan $(8,9,14,22)$, but these variations have several explanations. First, as the epidemic developed, more medical resources were invested in 


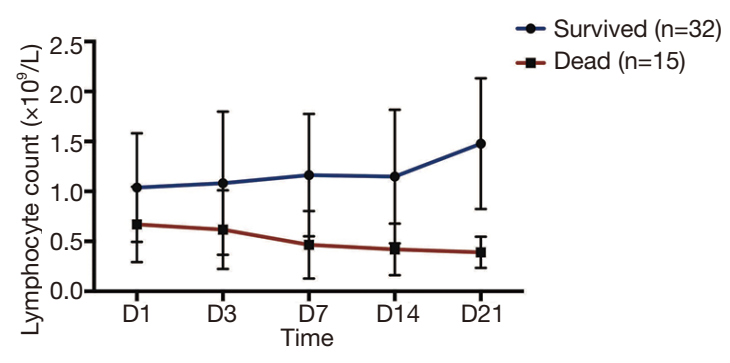

Figure 1 Temporal changes in lymphocyte count from illness onset in patients hospitalized with COVID-19. COVID-19, coronavirus disease 2019.

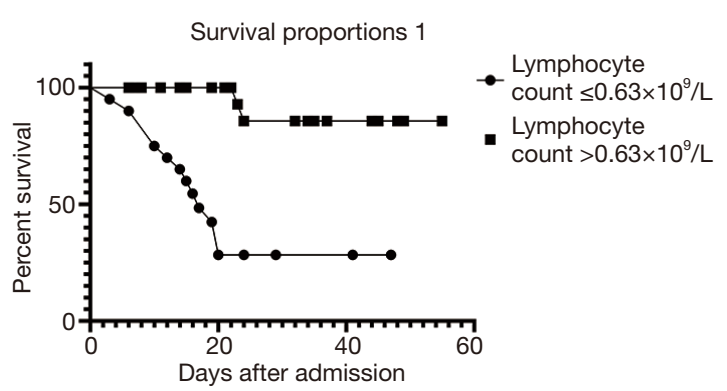

Figure 2 There was a significant difference in overall survival between the group with lymphocyte count $>0.63 \times 10^{9} / \mathrm{L}$ and the group with lymphocyte count $\leq 0.63 \times 10^{9} / \mathrm{L}$.

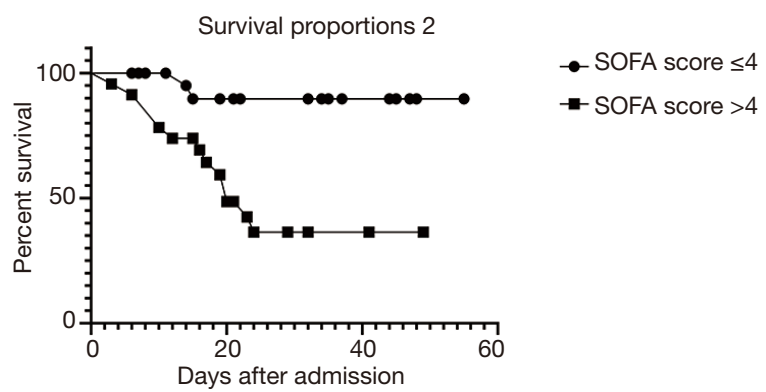

Figure 3 There was a significant difference in overall survival between the group with SOFA score $\leq 4$ and the group with SOFA score $>4$. COVID-19, coronavirus disease 2019; SOFA, Sequential Organ Failure Assessment.

Wuhan, more provisional ICUs were established, and the clinical capacity to treat patients improved greatly. Second, the median time from illness onset to admission was reduced. Third, the proportion of patients who required mechanical ventilation was lower in our ICU than in those of other studies, which may explain why the mortality rate in our study is similar to that reported for developed countries (10-12).

The death of patients with COVID-19 was found to be associated with older age in many studies $(6,14)$, including a large cohort of Italian COVID-19 patients in the ICU reported by Grasselli and colleagues (10). Age seemed not to be an independent risk factor in our study. That may be because the mean age of patients in our study was 70.55 years, which was notably older than that in the above studies.

Many comorbidities have previously been reported to indicate poor outcome of COVID-19 $(6,12,23)$, but the association between each specific comorbidity and death has not yet been fully explored, especially in the ICU. A study in Germany found that death was associated with preexisting lung disease, but not with any of other comorbidities in patients admitted to the ICU (11). In our ICU, comorbidities were present in $68.1 \%$ patients, similar to those seen by Grasselli et al. (10). Additionally, neither our investigation nor the one by Grasselli et al. found an independent association of comorbidities with mortality (10). This could be explained by the fact that patients admitted to the ICU had more comorbidities than other patients in the hospital (6).

SOFA and quick (q)SOFA scores are useful diagnostic tools for predicting the prognosis of adult inpatients with community-acquired pneumonia (CAP) and sepsis in the ICU $(24,25)$. A study by Asai et al. suggested that the combination of a qSOFA score $\geq 2$ and a SOFA score $\leq 4$ is a risk factor for 30-day mortality among CAP patients (26). Moreover, SOFA score criteria were found to be better than systemic inflammatory response syndrome criteria and the qSOFA score at predicting infection-related hospital mortality in ICU patients (27). For adult patients with COVID-19, a higher SOFA score at admission was also reported to be a risk factors for death (14), which was confirmed in our study. Non-survivors in our study had a mean SOFA of 7, which was higher than previously reported (14). Although SOFA scores may accurately evaluate the severity of disease in patients with COVID-19 in ICU, it will be necessary to conduct further prospective studies to assess the role of SOFA scores in predicting the prognosis of patients outside the ICU.

Our findings suggest that the lymphocytes count is a promising biomarker reflecting treatment efficacy and prognosis. The respiratory system and immune system are the main targets of SARS-CoV infections, with extensive consolidation of the lung, diffuse alveolar damage, and poor immunity identified as the main causes of death. An 
autopsy of patients with SARS identified a mass of necrosis in the splenic lymphoid tissue and localized necrosis of lymph nodes (28). In patients with COVID-19, SARSCoV-2 appears to mostly impact lymphocytes, especially T lymphocytes (29). Indeed, helper T cells, suppressor $\mathrm{T}$ cells and regulatory $\mathrm{T}$ cells were all below normal levels in reported COVID-19 cases, and more obviously damaged in severe cases, suggesting an imbalanced immunoreaction in the pathogenesis of COVID-19 (29). Zhou et al. reported a notably higher baseline lymphocyte count in survivors compared with non-survivors, and an improved lymphocyte count during the hospitalisation of survivors, while severe lymphopenia was observed until death in non-survivors (14). This is consistent with our current findings which revealed an association between lymphocyte count on admission and mortality, with patients with higher lymphocyte counts $\left(>0.63 \times 10^{9} / \mathrm{L}\right)$ on admission having a significantly better overall survival than those with lower lymphocyte counts $\left(\leq 0.63 \times 10^{9} / \mathrm{L}\right)$. The concept that lymphocytes are a potential therapeutic target deserves further investigation.

Our study has several limitations. First, it was a retrospective, single center study with a relatively small sample size, and laboratory tests for example serum ferritin and percentages of lymphocyte subsets percentage were not performed in all patients. Second, compared with patients in the published literature, our patients had a higher proportion of comorbidities. Additionally, some patients and their carers chose to stop the use of endotracheal intubation and mechanical ventilation, which may have affected the prognosis. Third, limited medical resources may have delayed hospitalization or admission for some patients in the early stages and several patients were transferred to other hospitals for comorbidities, which influenced the follow-up.

\section{Conclusions}

Old age and comorbidities are commonly seen in COVID-19 patients admitted to ICU. SOFA score and lymphocyte count on admission were found to be associated with prognosis of patients with COVID-19 in ICU. Higher SOFA score, and lower lymphocyte count were found to be independent risk factors of death. Lymphocyte count on admission may serve as a potential prognostic marker.

\section{Acknowledgments}

We thank Sarah Williams, PhD, from Liwen Bianji, Edanz
Editing China (www.liwenbianji.cn/ac), for editing the English text of a draft of this manuscript.

Funding: None.

\section{Footnote}

Reporting Checklist: The authors have completed the STROBE Checklist. Available at http://dx.doi. org/10.21037/apm-20-1575

Data Sharing Statement: Available at http://dx.doi. org/10.21037/apm-20-1575

Conflicts of Interest: All authors have completed the ICMJE uniform disclosure form (available at http://dx.doi. org/10.21037/apm-20-1575). The authors have no conflicts of interest to declare.

Ethical Statement: The authors are accountable for all aspects of the work in ensuring that questions related to the accuracy or integrity of any part of the work are appropriately investigated and resolved. The study was conducted in accordance with the Declaration of Helsinki (as revised in 2013). The study was approved by the ethics committee of Leishenshan Hospital, which was completely managed by the Renji Hospital (Shanghai Jiao Tong University School of Medicine) (NO.: [2020]023) and individuals consent for this retrospective analysis was waived.

Open Access Statement: This is an Open Access article distributed in accordance with the Creative Commons Attribution-NonCommercial-NoDerivs 4.0 International License (CC BY-NC-ND 4.0), which permits the noncommercial replication and distribution of the article with the strict proviso that no changes or edits are made and the original work is properly cited (including links to both the formal publication through the relevant DOI and the license). See: https://creativecommons.org/licenses/by-nc-nd/4.0/.

\section{References}

1. Phelan AL, Katz R, Gostin LO. The Novel Coronavirus Originating in Wuhan, China: Challenges for Global Health Governance. JAMA 2020;323:709-10.

2. World Health Organization. Coronavirus disease (COVID-19) outbreak. Available online: https:// www. who.int

3. WHO. WHO coronavirus disease (COVID-19) 
dashboard. 2020. Available online: https://covid19.who. int/

4. WHO. Report of the WHO-China Joint Mission on coronavirus disease 2019 (COVID-19). Feb 28, 2020. Available online: https://www.who.int/publicationsdetail/report-of-the-who-china-joint-mission-oncoronavirus-disease-2019-(covid-19) (accessed March 26, 2020).

5. Guan WJ, Ni ZY, Hu Y, et al. Clinical Characteristics of Coronavirus Disease 2019 in China. N Engl J Med 2020;382:1708-20.

6. Wang D, Hu B, Hu C, et al. Clinical Characteristics of 138 Hospitalized Patients With 2019 Novel CoronavirusInfected Pneumonia in Wuhan, China. JAMA 2020;323:1061-9.

7. Chen N, Zhou M, Dong X, et al. Epidemiological and clinical characteristics of 99 cases of 2019 novel coronavirus pneumonia in Wuhan, China: a descriptive study. Lancet 2020;395:507-13.

8. Yang X, Yu Y, Xu J, et al. Clinical course and outcomes of critically ill patients with SARS-CoV-2 pneumonia in Wuhan, China: a single-centered, retrospective, observational study. Lancet Respir Med 2020;8:475-81.

9. Cao J, Hu X, Cheng $\mathrm{W}$, et al. Clinical features and short-term outcomes of 18 patients with corona virus disease 2019 in intensive care unit. Intensive Care Med 2020;46:851-3.

10. Grasselli G, Zangrillo A, Zanella A, et al. Baseline Characteristics and Outcomes of 1591 Patients Infected With SARS-CoV-2 Admitted to ICUs of the Lombardy Region, Italy. JAMA 2020;323:1574-81.

11. Nachtigall I, Lenga P, Jóźwiak K, et al. Clinical course and factors associated with outcomes among 1904 patients hospitalized with COVID-19 in Germany: an observational study. Clin Microbiol Infect 2020;26:1663-9.

12. Docherty AB, Harrison EM, Green CA, et al. Features of 20133 UK patients in hospital with covid-19 using the ISARIC WHO Clinical Characterisation Protocol: prospective observational cohort study. BMJ 2020;369:m1985.

13. Wu C, Chen X, Cai Y, et al. Risk Factors Associated With Acute Respiratory Distress Syndrome and Death in Patients With Coronavirus Disease 2019 Pneumonia in Wuhan, China. JAMA Intern Med 2020;180:934-43.

14. Zhou F, Yu T, Du R, et al. Clinical course and risk factors for mortality of adult inpatients with COVID-19 in Wuhan, China: a retrospective cohort study. Lancet
2020;395:1054-62.

15. New coronavirus pneumonia prevention and control program (7th ed). 2020. Available online: http://www. nhc.gov.cn/yzygj

16. WHO. Clinical management of severe acute respiratory infection when novel coronavirus ( $\mathrm{nCoV}$ ) infection is suspected: interim guidance. January 28, 2020. Accessed March 5, 2020. Available online: https://www.who.int/ publications-detail/clinical-management-of-severe-acuterespiratory-infection-when-novel-coronavirus-(ncov)infection-is-suspected

17. Shankar-Hari M, Phillips GS, Levy ML, et al. Developing a New Definition and Assessing New Clinical Criteria for Septic Shock: For the Third International Consensus Definitions for Sepsis and Septic Shock (Sepsis-3). JAMA 2016;315:775-87.

18. Kidney Disease: Improving Global Outcomes (KDIGO) Acute Kidney Injury Work Group. KDIGO Clinical Practice Guideline for Acute Kidney Injury. Kidney Int Suppl 2012;2:1-138.

19. Huang C, Wang Y, Li X, et al. Clinical features of patients infected with 2019 novel coronavirus in Wuhan, China. Lancet 2020;395:497-506.

20. ARDS Definition Task Force, Ranieri VM, Rubenfeld GD, et al. Acute respiratory distress syndrome: the Berlin Definition. JAMA 2012;307:2526-2533.

21. Garner JS, Jarvis WR, Emori TG, et al. CDC definitions for nosocomial infections, 1988. Am J Infect Control 1988;16:128-40.

22. Chen T, Wu D, Chen H, et al. Clinical characteristics of 113 deceased patients with coronavirus disease 2019: retrospective study. BMJ 2020;368:m1091.

23. Giorgi Rossi P, Marino M, Formisano D, et al. Characteristics and outcomes of a cohort of COVID-19 patients in the Province of Reggio Emilia, Italy. PLoS One 2020;15:e0238281.

24. Raith EP, Udy AA, Bailey M, et al. Prognostic accuracy of the SOFA Score, SIRS criteria, and qsofa score for in-hospital mortality among adults with suspected infection admitted to the intensive care unit. JAMA 2017;317:290-300.

25. Ranzani OT, Prina E, Menéndez R, et al. New Sepsis Definition (Sepsis-3) and Community-acquired Pneumonia Mortality. A Validation and Clinical Decision-Making Study. Am J Respir Crit Care Med 2017;196:1287-97.

26. Asai N, Watanabe H, Shiota A, et al. Efficacy and accuracy of qSOFA and SOFA scores as prognostic 
tools for community-acquired and healthcare-associated pneumonia. Int J Infect Dis 2019;84:89-96.

27. Solligård E, Damås JK. SOFA criteria predict infectionrelated in-hospital mortality in ICU patients better than SIRS criteria and the qSOFA score. Evid Based Med 2017;22:211.

28. Ding Y, Wang H, Shen H, et al. The clinical pathology of severe acute respiratory syndrome (SARS): a report from China. J Pathol 2003;200:282-9.

29. Qin C, Zhou L, Hu Z, et al. Dysregulation of Immune Response in Patients With Coronavirus 2019 (COVID-19) in Wuhan, China. Clin Infect Dis 2020;71:762-8.

Cite this article as: Sai F, Liu X, Li L, Ye Y, Zhu C, Hang Y, Huang C, Tian L, Huang H, Xu X. Clinical characteristics and risk factors for mortality in patients with coronavirus disease 2019 in intensive care unit: a single-center, retrospective, observational study in China. Ann Palliat Med 2021;10(3):28592868. doi: 10.21037/apm-20-1575 